\title{
SEGUE, and the future of large scale surveys of the Galaxy
}

\author{
Timothy C. Beers ${ }^{1}$, Young Sun Lee ${ }^{1}$, and Daniela Carollo ${ }^{2}$ \\ ${ }^{1}$ Department of Physics \& Astronomy, CSCE: Center for the Study of Cosmic Evolution, and \\ JINA: Joint Institute for Nuclear Astrophysics, Michigan State University, \\ East Lansing, MI 48824, USA \\ email: beers@pa.msu.edu, lee@pa.msu.edu \\ ${ }^{2}$ Research School of Astronomy and Astrophysics, Australian National University, Mount \\ Stromlo Observatory, Cotter Road, Weston, ACT 2611, Australia, and INAF-Osservatorio \\ Astronomico di Torino, 10025 Pino Torinese, Italy \\ email: carollo@mso.anu.edu.au
}

\begin{abstract}
The Sloan Extension for Galactic Exploration and Understanding (SEGUE) has now been completed. This is one of three surveys that were executed as part of the first extension of the Sloan Digital Sky Survey (SDSS-II), which consist of LEGACY, SUPERNOVA SURVEY, and SEGUE. The SEGUE program has obtained over 3600 square degrees of ugriz imaging of the sky outside the original SDSS-I footprint. The regions of sky targeted for SEGUE imaging were primarily at lower Galactic latitudes $\left(|b|<35^{\circ}\right)$, in order to better sample the disk/halo interface of the Milky Way. SEGUE also obtained medium-resolution $(R=2000)$ spectroscopy, over the wavelength range $3800-9200 \AA$, for over 200,000 stars in 200 selected areas over the sky available from Apache Point, New Mexico. We discuss the determination of stellar atmospheric parameters $\left(\mathrm{T}_{\mathrm{eff}}, \log \mathrm{g}\right.$, and $\left.[\mathrm{Fe} / \mathrm{H}]\right)$ for these stars, and highlight several of the scientific results obtained to date. The proposed second extension of SDSS, known as SDSS-III, will include SEGUE-2, a program to roughly double the numbers of stars with available spectroscopy, as well as APOGEE, a program to obtain high-resolution $(R=20000)$ near-IR spectroscopy for over 100,000 stars in the disk, bulge and halo populations of the Galaxy. Other massive spectroscopic surveys of interest to Galactic science are also briefly discussed.
\end{abstract}

Keywords. Astronomical data bases: surveys; techniques: spectroscopic; methods: data analysis; stars: fundamental parameters; Galaxy: disk, halo

\section{Introduction}

We are now firmly entrenched in the era of massive photometric and spectroscopic surveys of the stellar populations of the Galaxy, as highlighted by the Sloan Digital Sky Survey (SDSS-I), and its first extension, SDSS-II, as well as by the contemporaneous survey RAVE. These surveys, and others that are planned for the near future, promise to completely revolutionize our understanding of the formation and evolution of the Milky Way, and provide new insight into the formation of the chemical elements.

Here we summarize the status of the SEGUE (Sloan Extension for Galactic Exploration and Understanding) program, executed as part of SDSS-II, and foreshadow the goals of SEGUE-2, to be carried out during the proposed next extension of SDSS, known as SDSS-III. We also briefly mention several additional ongoing high-resolution spectroscopic surveys, as well as a number of other massive surveys planned for the near (APOGEE, LAMOST) and the more distant future (WFMOS). 


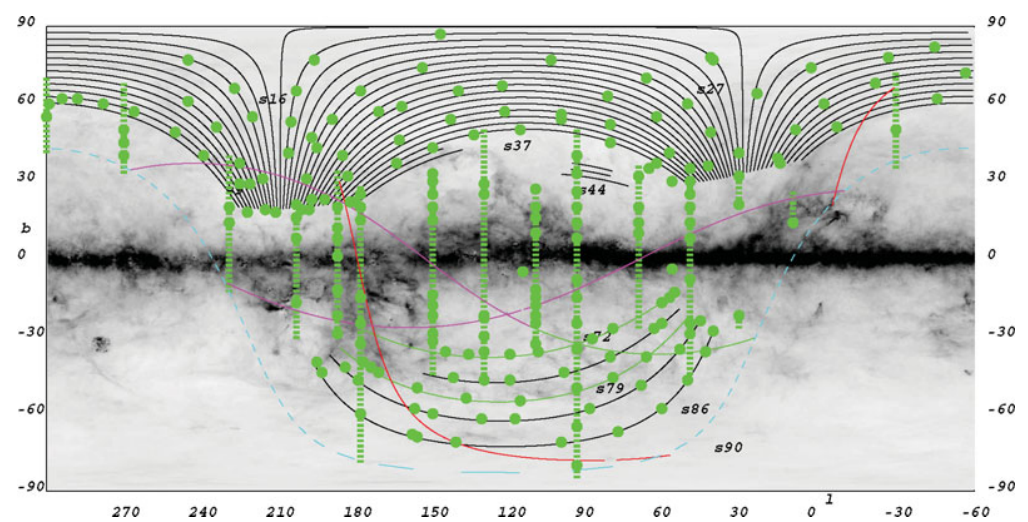

Figure 1. Map of the imaging and spectroscopic coverage obtained during the SDSS/SEGUE program. The black lines correspond to regions imaged during SDSS-I, while the colored lines indicate regions of SEGUE imaging. The green filled dots indicate lines of sight where SEGUE obtained over 1200 individual spectra (in two spectroscopic plug-plates, a bright plate and a faint plate). The image of the Milky Way is derived from the dust maps of Schlegel et al. (1998).

\section{SEGUE}

The SEGUE survey took its last data in June, 2008. Over 3600 square degrees of new imaging was obtained, and a total of over 400 spectroscopic plug-plates were obtained, covering some 200 lines of sight probing directions of interest for exploration of the thick disk and halo populations of the Milky Way, from $0.5 \mathrm{kpc}$ to almost $100 \mathrm{kpc}$ from the Sun (see Fig. 1). The medium-resolution $(R=2000)$ spectroscopy obtained for roughly 240,000 program stars (in the magnitude range $14.0<g<20.5$ ), in combination with the ugriz photometry from the imaging, form the inputs for determinations of radial velocities (accurate to between 5 and $25 \mathrm{~km} / \mathrm{s}$, depending on the color of the star and the $\mathrm{S} / \mathrm{N}$ of the spectrum) and, most crucially, the fundamental stellar atmospheric parameters $\left(\mathrm{T}_{\text {eff }}, \log \mathrm{g}\right.$, and $\left.[\mathrm{Fe} / \mathrm{H}]\right)$ for the majority of the program objects. The full data set (and derived parameters) is planned to be released to the public in October, 2008.

The atmospheric parameters are derived from a set of techniques, based on a number of different calibrations, in an effort to provide robust determinations that remain valid over the large range of parameter space and S/N explored by SEGUE. These approaches, which collectively are applied by the SEGUE Stellar Parameter Pipeline (SSPP), include techniques for finding the minimum distance (parameterized in various ways) between observed spectra and grids of synthetic spectra (e.g., Allende Prieto et al. 2006), non-linear regression models (e.g., Re Fiorentin et al. 2007, and references therein), correlations between broadband colors and the strength of prominent metallic lines, such as the CaII K line (Beers et al. 1999), auto-correlation analysis of a stellar spectrum (Beers et al. 1999, and references therein), obtaining fits of spectral lines (or summed line indices) as a function of broadband colors (Wilhelm et al. 1999), or the behavior of the CaII triplet lines as a function of broadband color (Cenarro et al. 2001).

Each of the methods employed by the SSPP exhibit optimal behavior over restricted temperature and metallicity ranges; outside of these regions they are often un-calibrated, suffer from saturation of the metallic lines used in their estimates at high metallicity or low temperatures, or lose efficacy due to the weakening of metallic species at low metallicity or high temperatures. The techniques that make use of specific spectral features 
are susceptible to other problems, e.g., the presence of emission in the core of the CaII K line for chromospherically active stars, or poor telluric line subtraction in the region of the CaII triplet. Because SDSS stellar spectra cover most of the entire optical wavelength range, one can apply several approaches, using different wavelength regions, in order to glean optimal information on stellar parameters. The combination of multiple techniques results in estimates of stellar parameters that are more robust over a much wider range of $\mathrm{T}_{\text {eff }}, \log \mathrm{g}$, and $[\mathrm{Fe} / \mathrm{H}]$ than those that might be produced by individual methods.

Details of these procedures and tests of the validity of the resulting parameter estimates are presented in a series of three papers, Lee et al. (2008a), Lee et al. (2008b), and Allende Prieto et al. (2008), to which the interested reader is referred.

The precision of the parameter estimates varies with the $\mathrm{S} / \mathrm{N}$ of the spectra. At the median S/N of the SEGUE spectra (roughly 20/1), the estimates have typical errors of $\delta \mathrm{T}_{\text {eff }}=150 \mathrm{~K}, \delta \log \mathrm{g}=0.30 \mathrm{dex}$, and $\delta[\mathrm{Fe} / \mathrm{H}]=0.25$ dex, respectively. Tests on the accuracy of the atmospheric parameter estimates indicate that there exist negligible zero-point offsets over the majority of the parameter space.

\section{Summary of early results from SEGUE}

Although the final data set has only just been obtained, numerous scientific results from SEGUE have already emerged. A few of these include:

- The identification of ultra low luminosity dwarf spheroidals, and halo debris streams (Belokurov et al. 2006, Zucker et al. 2006). It has been argued, based on new highresolution spectroscopic estimates of $[\mathrm{Fe} / \mathrm{H}]$ (see Kirby et al. 2008), that these newly discovered dwarfs indeed include substantial numbers of stars with $[\mathrm{Fe} / \mathrm{H}]<-3.0$, strengthening the possible association of the formation of the (outer) halo with the accretion and destruction of similar objects in the past.

- Confirmation of the inner/outer halo structure of the Milky Way (Carollo et al. 2007). Although it had been long speculated that the halo of the Milky Way might be structurally complex, and comprise more than a single stellar population, the calibration stars from SDSS/SEGUE have been used to demonstrate convincingly that the halo is indeed clearly divisible into two broadly overlapping structural components, an inner and an outer halo, which exhibit different spatial density profiles, stellar orbits, and stellar metallicities. While the inner halo has a modest net prograde rotation, the outer halo exhibits a net retrograde rotation and a peak metallicity three times lower $([\mathrm{Fe} / \mathrm{H}]$ $=-2.2)$ than that of the inner halo $([\mathrm{Fe} / \mathrm{H}]=-1.6)$. These properties indicate that the individual halo components likely formed in fundamentally different ways, through successive dissipational (inner) and dissipationless (outer) mergers and tidal disruption of proto-Galactic clumps. Work is now in progress (Carollo et al., in prep.) to derive the velocity ellipsoids and metallicity distribution functions of the individual populations. See Fig. 2.

- Measurement of a new (lower) mass for the Galaxy (Xue et al. 2008). These authors isolated a nearly pure sample of some 2400 blue horizontal-branch stars with distances up to $60 \mathrm{kpc}$ from the Galactic center, and compared their observed radial velocities with cosmologically motivated galaxy formation scenarios to obtain estimates of the Milky Way's circular velocity curve, implying $\mathrm{M}(<60 \mathrm{kpc})=4.0 \pm 0.7 \times 10^{11} \mathrm{M}_{\odot}$. The associated virial mass of the dark halo of the Milky Way is estimated to be $\mathrm{M}_{\mathrm{vir}}=$ $0.93 \pm 0.25 \times 10^{12} \mathrm{M}_{\odot}$, which is lower than many previous estimates. This estimate implies that nearly $40 \%$ of the baryons within the virial radius of the Milky Way's dark matter halo reside in the stellar components of our Galaxy. 

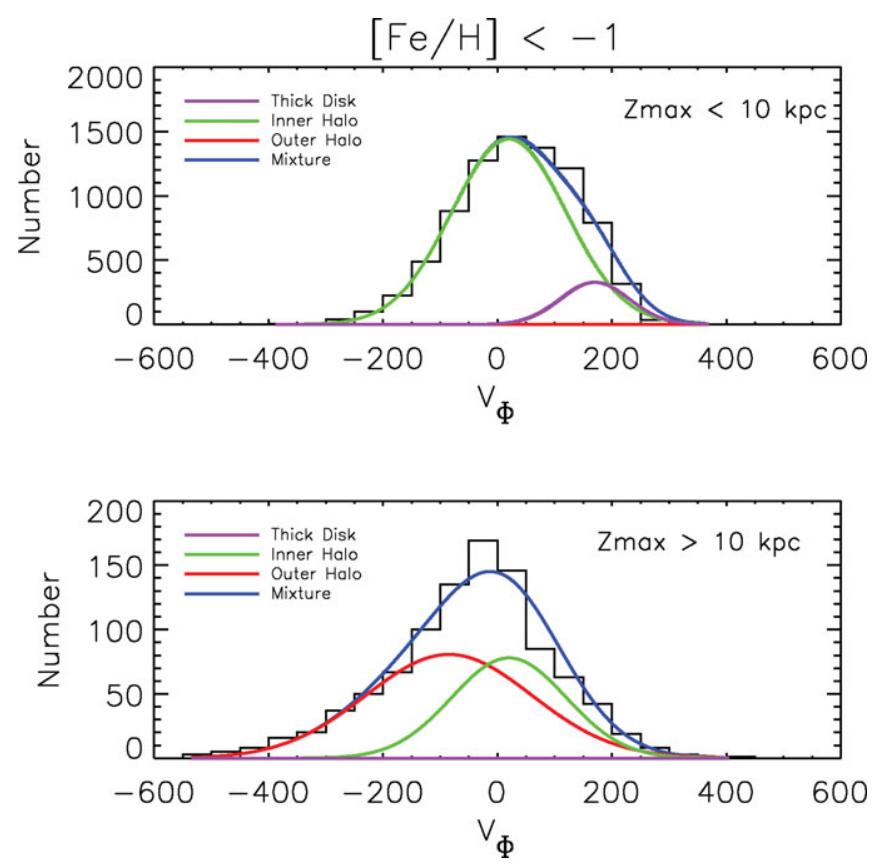

Figure 2. An example maximum-likelihood decomposition of the contribution of thick disk, inner-halo, and outer-halo populations to the overall observed distribution of $\mathrm{V}_{\Phi}$, the rotation with respect to the Galactic center, based on an analysis of the SDSS/SEGUE calibration stars from DR-6 (Adelman-McCarthy et al. 2008). All stars in the figure have $[\mathrm{Fe} / \mathrm{H}]<-1.0$. The upper panel applies to stars with $Z_{\max }<10 \mathrm{kpc}$; the lower panel applies to stars with $\mathrm{Z}_{\max }>$ $10 \mathrm{kpc}$, where $Z_{\max }$ refers to the maximum distance from the Galactic plane reached by an individual star during the course of its orbit. Note that the inner-halo population completely dominates the observed distribution in the upper panel, while a higher dispersion, net retrograde outer-halo population is required to account for the extended low velocity tail in the lower panel.

- Construction of a metallicity map for millions of stars in the disk and halo populations of the Milky Way (Ivezic et al. 2008). Based on the spectroscopically calibrated stars from SDSS/SEGUE, these authors have derived photometric estimates of $[\mathrm{Fe} / \mathrm{H}]$ for some 2.5 million F-type stars in the color range $0.2<g-r<0.4$, with accuracies only slightly less than the spectroscopic determinations, and exploring distances up to 9 kpc from the Sun. Such a technique will be used in the future, e.g., with data from LSST and SkyMapper, to obtain metallicity maps of the halo of the Galaxy out to 100,000 kpc, with substantially improved accuracy. See Fig. 3.

- Measurement of $[\alpha / \mathrm{Fe}]$ ratios for tens of thousands of stars (Lee et al., in prep.). As part of his $\mathrm{PhD}$ thesis, Lee has calibrated techniques to obtain estimates of the $[\alpha / \mathrm{Fe}]$ ratio for high-S/N SDSS/SEGUE spectra ( $\mathrm{S} / \mathrm{N}>25 / 1)$. The method is precise to on the order of $\delta[\alpha / \mathrm{Fe}]=0.1 \mathrm{dex}$, which is sufficient to provide information concerning the nature of the sub-Galactic fragments (such as their mass distributions) involved with the assembly of the thick disk, and the inner- and outer-halo populations. See Fig. 4.

- The identification of over $100,000 \mathrm{~F}-\mathrm{G}-\mathrm{K}$ stars with $[\mathrm{Fe} / \mathrm{H}]<-1.0$, over 15,000 with $[\mathrm{Fe} / \mathrm{H}]<-2.0$, and several hundred with $[\mathrm{Fe} / \mathrm{H}]<-3.0$ (Beers et al., in prep.)

- The identification of many thousands of Carbon-Enhanced Metal-Poor (CEMP) Stars (Sivarani et al., in prep.) 


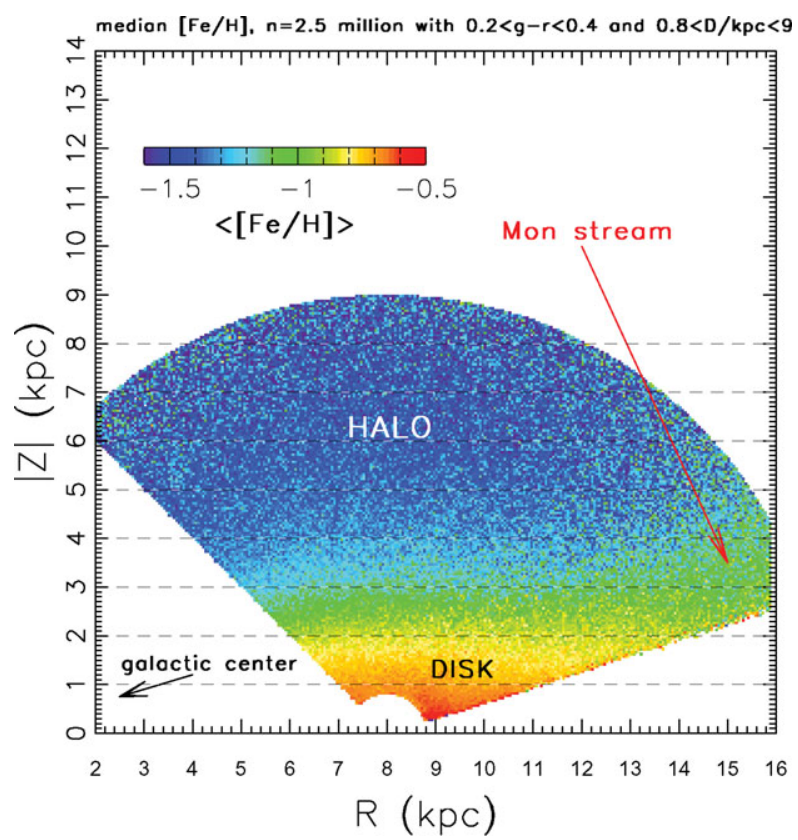

Figure 3. Photometric metallicities for 2.5 million blue F-type stars, based on the calibration described by Ivezic et al. 2008. The median occupancy of the 40,000 pixels shown in this map is 33 stars, with a minimum of 5 stars per pixel. The color-coded metallicities clearly indicate the presence of the disk, thick-disk, and inner-halo populations of the Galaxy. The presence of the Monoceros stream in the region close to the disk plane is indicated.

\section{Future surveys}

SEGUE-2 is one of four surveys planned as part of the proposed next extension to the SDSS, known as SDSS-III. SEGUE-2 will use the existing SDSS spectrograph to obtain medium-resolution spectra for roughly an additional 200,000 stars, during a period of one year starting in August, 2008. Building on what has been learned from SEGUE, SEGUE2 will specifically target stars (in a variety of categories) that explore the transition from the inner to the outer halo, concentrating on stars at the main-sequence turnoff, distant giants and horizontal-branch stars, and on stars selected to be likely low-metallicity objects.

There exist a number of large high-resolution spectroscopic surveys that are just now getting underway, which should greatly enlarge the numbers of very metal-poor (VMP, $[\mathrm{Fe} / \mathrm{H}]<-2.0)$ stars with available elemental abundance information. The Chemical Abundances of Halo Stars (CASH) survey is making use of the Hobby-Eberly Telescope to obtain moderately high-resolution $(R=15000)$ spectroscopy for up to $1,000 \mathrm{VMP}$ stars identified during the course of the HK-I, HK-II, and HES efforts, with a large number of additional stars from SDSS/SEGUE. Aoki and collaborators have recently been awarded Key Project status on the Subaru Telescope in order to obtain $R=50000$ spectroscopic observations for up to $200 \mathrm{VMP}$ stars, the majority of which will be drawn from SDSS/SEGUE targets. Both of these surveys have as one of their primary aims to test for the existence (or not) of chemical signatures that might be associated with the inner/outer halo dichotomy reported by Carollo et al. (2007).

The Apache Point Observatory Galactic Evolution Experiment (APOGEE), another survey to be conducted as part of SDSS-III, will use high-resolution $(R=20000)$, high 


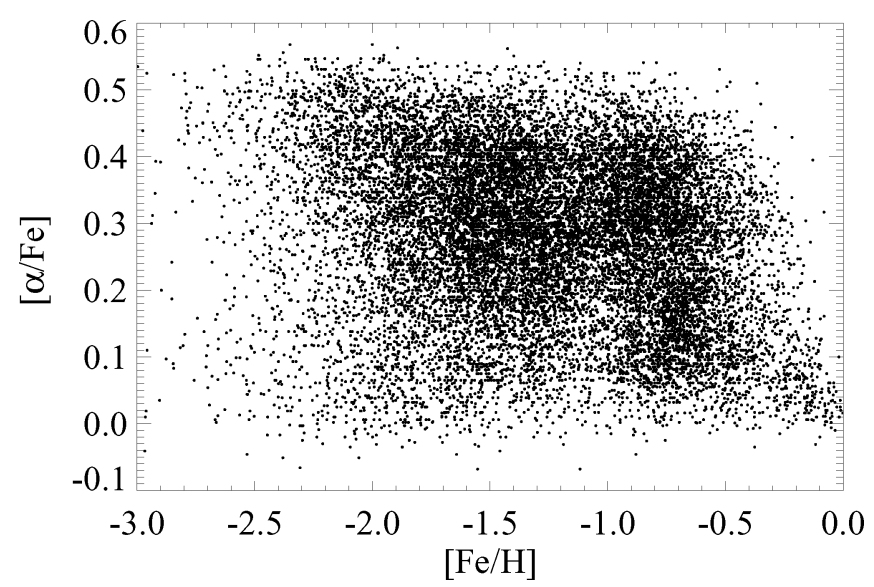

Figure 4. Distribution of $[\alpha / \mathrm{Fe}]$ for over 18,000 stars from SDSS/SEGUE, as a function of $[\mathrm{Fe} / \mathrm{H}]$. There is an apparent bifurcation of this distribution at low metallicity, into predominantly high alpha abundances and low alpha abundances. The full implications of these observations will require detailed modeling to better understand.

signal-to-noise ratio $(\mathrm{S} / \mathrm{N}=100 / 1)$, H-band $(1.6 \mu \mathrm{m})$ spectroscopy to penetrate the dust that obscures the inner Galaxy from our view, observing 100,000 red giant stars across the full range of the Galactic bulge, bar, disk, and halos. The high spectral resolution of APOGEE will allow element-by-element measurements of chemical abundances, which can be used to reconstruct the history of star formation that produced these elements. Together, SEGUE-2 and APOGEE will provide a picture of the Milky Way that is unprecedented in scope, richness, and detail. The combined data set will play a central role in "near-field cosmology" tests of galaxy formation physics and the small scale distribution of dark matter.

The Large Area Multi-Object Spectroscopy Telescope (LAMOST) project, funded by the Chinese National Academy of Science, will employ a massive 4000 fiber system mounted on a wide-field $4 \mathrm{~m}$ meridian Schmidt telescope. It is expected that this ambitious project will obtain up to several million medium-resolution $(R=2000)$ spectroscopic observations of stars in the Milky Way and Local Group galaxies. With the planned addition of $R=5000 / 10000$ gratings, follow-up observations at moderately high spectral resolution will also become possible.

Of course, we all look forward to the possible execution of the WFMOS (Wide Field Multi-Object Spectrograph) survey of up to one million stars at resolving power $R=$ 50000. Currently, this Gemini instrument is expected to be mounted on the prime focus of the Subaru telescope, in order to take advantage of its wide field of view. The hope, and expectation, is that this survey will finally reveal the rich set of elemental abundances for stars that probe the entire history of chemical evolution throughout the Galaxy.

\section{Acknowledgements}

Funding for the SDSS and SDSS-II has been provided by the Alfred P. Sloan Foundation, the Participating Institutions, the National Science Foundation, the U.S. Department of Energy, the National Aeronautics and Space Administration, the Japanese Monbukagakusho, the Max Planck Society, and the Higher Education Funding Council for England. The SDSS Web Site is http://www.sdss.org/. 
This work received partial support from grants AST 07-07776 and PHY 02-15783; Physics Frontier Center / Joint Institute for Nuclear Astrophysics (JINA), awarded by the US National Science Foundation.

\section{References}

Adelman-McCarthy, J. K., Agüeros, M. A., Allah, S. S, Allende Prieto, C., \& Anderson, K. S. J., et al. 2008, ApJS, 175, 297

Allende Prieto, C., Beers, T. C., Wilhelm, R., Newberg, H. J., \& Rockosi, C. M., et al. 2006, ApJ, 636, 804

Allende Prieto, C., Sivarani, T., Beers, T. C., Lee, Y. S., \& Koesterke, L. et al. 2008, AJ, in press (arXiv:0710.5780)

Beers, T. C., Rossi, S., Norris, J. E., Ryan, S. G., \& Shefler, T. 1999, AJ, 117, 981

Belokurov, V., Zucker, D. B., Evans, N. W., Gilmore, G., \& Vidrih, S., et al. 2006, ApJ, 642, L137

Carollo, D., Beers, T. C., Lee, Y. S., Chiba, M., \& Norris, J. E., et al. 2007, Nature, 450, 1020

Cenarro, A. J., Cardiel, N, Gorgas, J., Peletier, R. F., Vazdekis, A., \& Prada, F. 2001, MNRAS, 326,959

Ivezic, Z., Sesar, B., Juric, M., Bond, N., \& Dalcanton, J., et al. 2008, ApJ, in press (arXiv:0804.3850)

Kirby, E. N., Simon, J. D., Geha, M., Guhathakurta, P., \& Frebel, A. 2008, ApJ, submitted (arXiv:0807.1925)

Lee, Y. S., Beers, T. C., Sivarani, T., Allende Prieto, C., \& Koesterke, L., et al. 2008, AJ, in press (arXiv:0710.5645)

Lee, Y. S., Beers, T. C., Sivarani, T., Johnson, J. A., \& An, D., et al. 2008, AJ, in press (arXiv:0710.5778)

Re Fiorentin, P., Bailer-Jones, C. A. L., Lee, Y. S., Beers, T. C., \& Sivarani, T., et al. 2007, $A \mathscr{E} A, 467,1373$

Schlegel, D. J., Finkbeiner, D. P., \& Davis, M. 1998, ApJ, 500, 525

Wilhelm, R., Beers, T. C., \& Gray, R. O. 1999, AJ, 117, 2308

Xue, X.-X., Rix, H.-W., Zhao, G., Re Fiorentin, P., \& Naab, T., et al. 2008, ApJ, in press (arXiv:0801.1232)

Zucker, D. B., Belokurov, V., Evans, N. W., Wilkinson, M. I., Irwin, M. J., et al. 2006, ApJ, 643, L103 


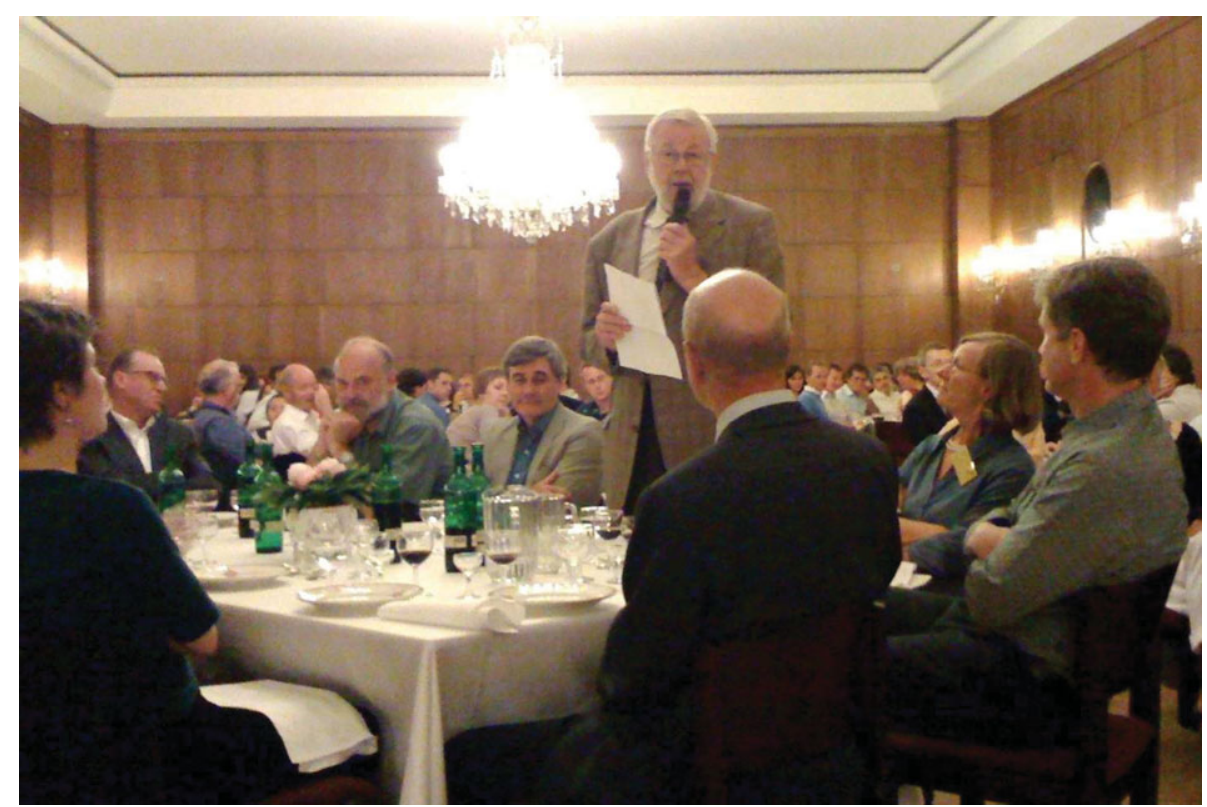

Ole Strömgren giving his speech at the closing dinner. From the left around the table: Line Kragh, Flemming Besenbacher, John Renner Hansen, Gerry Gilmore, Ole Strömgren, Birgitta Nordström, Joss Bland-Hawthorn, and Johannes Andersen (from back). Photo: Lars Buchhave.

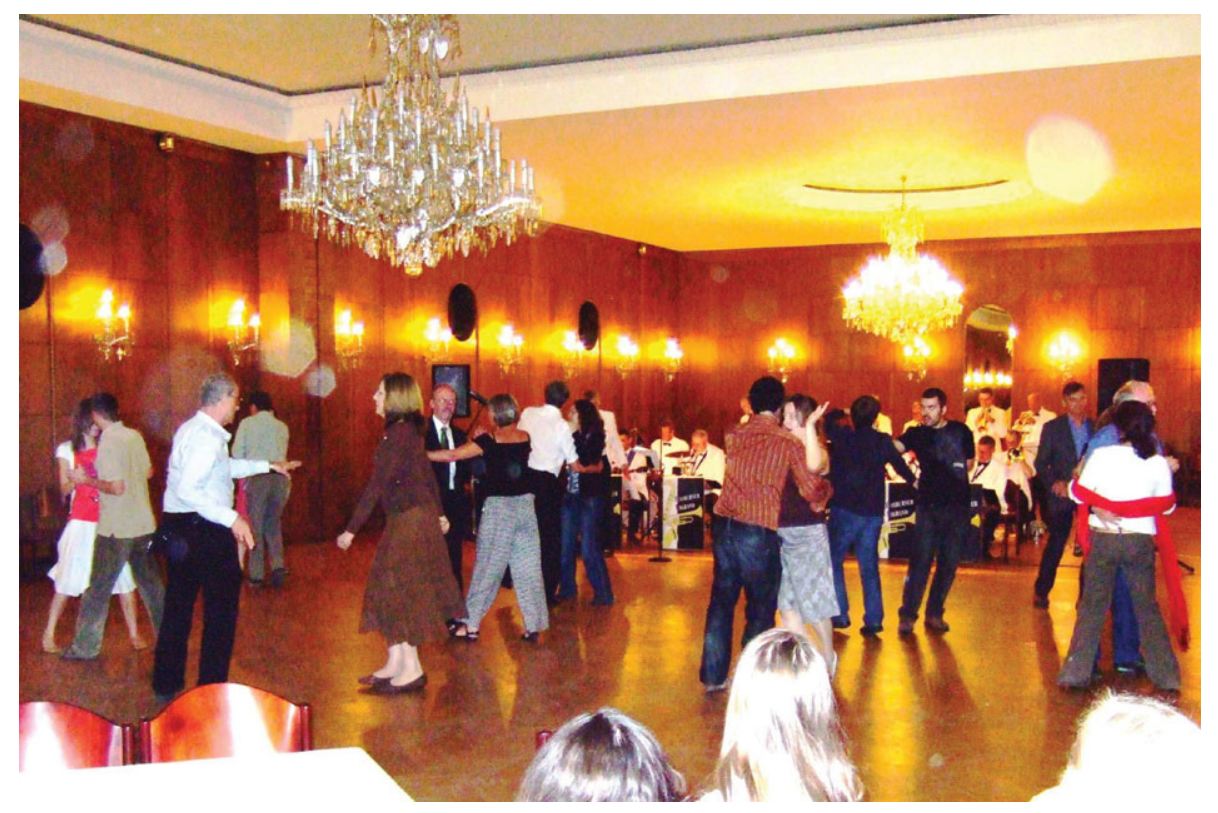

Enthusiastic participants dancing to the live Big-Band after dinner. 